\title{
Review of: "Hyperspectral Camera System"
}

\author{
Shengliang $\mathrm{Pu}^{1}$ \\ 1 Wuhan University
}

Potential competing interests: The author(s) declared that no potential competing interests exist.

It captures a large number of continuous spectral bands which have high spectral resolution and rich spectral information. 